Li, L., Zhang, M. \& Holman, C.D.J. (2013). Population versus hospital controls in the assessment of dietary intake of isoflavone for case-control studies on cancers in China. Nutrition and Cancer, 65(3), 390-397.

Copyright $@ 2013$, Taylor \& Francis Group, LLC

This is pre-copy-editing, author-produced version of an article accepted for publication in Nutrition and Cancer, following peer review. The definitive published version (see citation above) is located on the article abstract page of the publisher, Taylor and Francis.

This version was made available in the UWA Research Repository on 26 March 2014, in compliance with the publisher's policies on archiving in institutional repositories.

This is an Author's Accepted Manuscript of an article (Li, L., Zhang, M. \& Holman, C.D.J . (2013). Population versus hospital controls in the assessment of dietary intake of isoflavone for case-control studies on cancers in China. Nutrition and Cancer, 65(3), 390-397. ) as published in the Nutrition and Cancer, on 26 March 2013 (Copyright $\subset$ Taylor \& Francis). Available online at:

http://www.tandfonline.com/10.1080/01635581.2013.767915 
HNUC \#767915, VOL 65, ISS 3

\title{
Population Versus Hospital Controls in the Assessment of Dietary Intake of Isoflavone for Case-Control Studies on Cancers in China
}

\author{
Lin Li, Min Zhang, and C. D'Arcy J. Holman
}

\section{QUERY SHEET}

This page lists questions we have about your paper. The numbers displayed at left can be found in the text of the paper for reference. In addition, please review your paper as a whole for correctness.

Q1. Au: Please provide date.

Q2. Au: Please verify correspondence author and address, and add phone, fax, and email.

Q3. Au: Please add running head of 50 characters or less.

Q4. Au: Please provide last column head.

Q5. Au: The sentence beginning "The discrepancies between this study and..." is unclear. Please clarify.

Q6. Au: Journal style is to list 5 authors before use of "et al."

Q7. Au: In Ref. 23, journal style is to list first 5 authors beforse use of et al.

\section{TABLE OF CONTENTS LISTING}

The table of contents for the journal will list your paper exactly as it appears below:

Population Versus Hospital Controls in the Assessment of Dietary Intake of Isoflavone for Case-Control Studies on Cancers in China

Lin Li, Min Zhang, and C. D'Arcy J. Holman 


\title{
Population Versus Hospital Controls in the Assessment of Dietary Intake of Isoflavone for Case-Control Studies on Cancers in China
}

\author{
Lin Li, Min Zhang, and C. D'Arcy J. Holman \\ 5 School of Population Health, The University of Western Australia, Crawley, Australia
}

\begin{abstract}
To assess dietary isoflavone intake between population and hospital outpatient controls and examine if cancer risks estimated for isoflavone using hospital outpatient controls would be different 10 from those using population controls. Three parallel case-control studies on leukemia, breast, and colorectal cancers in China in 2009-2010 were conducted, using population and hospital outpatient controls to separately match 560 incident cases at a 1:1 ratio. A validated food frequency questionnaire was administered by 15 face-to-face interview. Conditional logistic regression analysis was used to estimate odds ratios (ORs) and $95 \%$ confidence intervals (CIs). The 2 control groups had closely similar distributions of dietary isoflavone intake. Risk estimates for breast cancers were adjusted ORs $(95 \% \mathrm{CI})$ of $0.39(0.23-0.66)$ and $0.31(0.18-0.55)$ for 20 daidzein, $0.35(0.20-0.61)$ and $0.28(0.16-0.52)$ for genistein, 0.66 (0.41-1.08) and $0.53(0.32-0.88)$ for glycitein, and $0.53(0.33-0.85)$ and $0.43(0.26-0.71)$ for total isoflavone using hospital outpatient and population controls respectively. The study found that hospital outpatient controls were comparable to population controls

25 in measured dietary intake of isoflavone in the Chinese hospital setting.
\end{abstract}

\section{INTRODUCTION}

The selection of an appropriate control group in case-control studies is widely acknowledged as a crucial methodological issue, because inappropriate selection can present a serious challenge to the internal validity of case-control study results. The control group is important because it provides a basis for comparison by representing what is normal or expected. In the hospital setting, the choice between hospital and population controls has generated controversy among epidemiologists due to tradeoffs between different potential sources of selection bias, response bias, and recall bias, as well as the most effective use of study resources (1-5).

Submitted xxx; accepted in final form xxx.

Address correspondence to Lin Li, School of Population Health, The University of Western Australia, 35 Stirling Highway, Crawley, WA 6009, Australia.
There are concerns that hospital controls, especially those with other diseases in most Western countries where general practitioners (GPs) serve as gatekeepers for access to the rest of the healthcare system, the exposure may be different from that in the source population, given that many causes of hospitalization are associated with or may cause selective dietary patterns. Therefore, hospital controls may fail to provide an unbiased sample of the population at risk with respect to exposure status in case-control studies (1-5).

China lacked an effective primary care system until recently, and thus most people sought medical care in hospitals, especially the large hospital in big cities, because these facilities are believed to provide the best care and China has no gatekeeper system (6). Outpatients readily see physicians as self-referred outpatients at hospital outpatient departments for diagnostic tests and treatment, most of whom do not have any specific disease. Therefore, the challenges to validity posed by hospital outpatient control selection from outpatient departments are less burdensome in China than in Western countries.

There are very few epidemiological studies comparing hospital controls with population controls, although the published theory on epidemiological texts is extensive. In particular, little information exists on differences between population and hospital outpatient controls selected from outpatient department in the hospital setting in non-Western countries.

Given that we have compared differences between population and hospital outpatient controls on demographic characteristics and lifestyle factors (7), and soy foods are widely consumed in Asian countries and are an almost exclusive dietary source of isoflavone (8-10), our previous case-control studies of isoflavone intake and cancer in China had used hospital controls. Therefore, this study aimed to compare isoflavone intake between hospital outpatient controls and alternative population controls to determine if there was any difference of practical importance, and to examine if inferences drawn from estimates of effects of dietary isoflavone on cancer risk using hospital outpatient controls would be different from those using population controls in the Chinese hospital setting. 


\section{METHODS}

Detailed study methods were published elsewhere (7). The 80 study, as a validation component of 3 large case-control studies of malignancies, was conducted in Shenyang, the capital city of Liaoning Province, Northeast China. In August 2009 to July 2010, 560 incident cases with a primary diagnosis of leukemia, or breast cancer, or colorectal cancer were identified

85 from histopathology and haematology records at the First Hospital of China Medical University, a public teaching hospital with 2249 beds, around 32,000 inpatients annually and 3000 outpatients daily. The eligible cases were permanent residents of urban Shenyang aged 18 to 85 years. During the study period,

90560 population controls and 560 hospital controls were selected from Shenyang residents and from outpatients at the same hospital to separately match cases in a 1:1 ratio. Most of controls were interviewed within 3 mo after cases were interviewed.

The methods of recruiting population controls were similar

95 to those used in case-control studies in Shanghai, China $(11,12)$. Population household registries, which kept records of all permanent residents in urban Shenyang, were used to select controls from the five metropolitan districts in Shenyang, namely Heping, Shenhe, Dadong, Huanggu, and Tiexi. With the assis-

100 tance of the local community councils, residents who lived at their registered address during the study period were randomly selected from household registry rolls. Residents were eligible as population controls if they matched with individual cases of an updated list by gender and year-of-birth quinquennium on a given selection day.

Hospital controls were drawn from the population of patients in the hospital (13). A systematic selection process used in our previous studies was adopted for hospital control recruitment (14). They were selected from outpatients who attended the

110 Medical Examination Centre of The Outpatient Department at the same hospital as cases and were permanent residents of urban Shenyang. The eligible hospital controls were those without any malignancy after they had consulted their doctors. Each hospital control was selected as the first attendee on a given selection

115 day to match the next case on a daily updated list of cases by sex and 5-yr age group. Hospital outpatients were excluded as a control if they were not matched to their corresponding cases by gender, year-of-birth quinquennium, and living areas, and if they had a diagnosis of any malignancy before or after recruit-

120 ment. The project protocol had received ethics approval from both the Human Research Ethics Committee of The University of Western Australia and the First Hospital of China Medical University authority.

A validated questionnaire was used to collect the informa125 tion by personal interview on (a) demographic and lifestyle characteristics (e.g., residential area, education, weight and height, and physical activity); (b) habitual dietary intake 1-yr prior to diagnosis, assessed by a 119-item food frequency questionnaire (FFQ), including 9 items on soy and soy-based foods

130 (dry soybean, fresh green soybean, tofu, dried or pressed bean curd, fried bean curd, fermented bean curd, soybean milk, skin of soybean milk, and soybean sprouts); and (c) factors relevant to hormonal status including menstrual history and menopausal status, reproductive and lactation history, hormone replacement treatment, history of use of oral contraceptives, other factors relevant to hormonal status, and family history of malignancy (15-18). Interviews were conducted by the first author and trained local research assistants and usually took 30-40 minutes.

Dietary intake of isoflavone were assessed using the FFQ to measure soy and soy-based foods. This quantitative FFQ had been used in our previous cancer research (15). This instrument was originally modified from one used for studying cancers in Shanghai to ensure cultural relevance (16). The frequencies of food intake were assigned to 9 categories of never or hardly ever, once/mo, 2 to 3 times/mo, once/wk, 2 to 3 times/wk, 4 to 6 times/wk, once/day, twice/day, and $\geq 3$ times/day. The quantitative variables were measured using the liang (equivalent to $50 \mathrm{~g}$ ), a Chinese unit very familiar to the participants. If there was any recent change in habits, only information on the habits before the change was used in data analysis. The validity and 150 reliability of the FFQ were assessed in previous studies $(17,18)$. The reproducibility of mean daily intake of soy and its products was indicated by an intraclass correlation coefficient of 0.78 for all soy foods combined (17).

All data were checked for completeness at the end of each 155 interview. The data were coded and analyzed using the SPSS version 18.0. The frequency and quantity variables derived from the FFQ were converted into daily food consumption after adjustments for the edible portions of foods, cooking methods used, seasonal factors, and market availability (19). These sorts of adjustments were adapted from Chinese nutrient database level that includes foods represent what is actually available and consumed (20). Total energy and nutrient intake were estimated using Chinese Food Composition Tables (20). Daily intake in $\mathrm{mg}$ of daidzein, genistein, glycitein, and total isoflavone were calculated based on daily food consumption using a recent isoflavone database from the USDA (21). Levels of isoflavone intake were divided into quartiles based on the empirical distributions in the population controls, with the lowest quartile being used in regression analyses as the reference category. Presence of tobacco smoking was defined as a total of 20 packs of cigarettes or more in a lifetime. Participants' self-reported height in meters and weight in kilograms were used to calculate body mass index (BMI; weight/height ${ }^{2}$ ).

Selected demographic characteristics and lifestyle factors of 175 two control groups were first compared separately in males and females using a $t$-test for continuous variables and chi-square test for categorical variables. Univariate analysis was undertaken to screen potential explanatory variables for subsequent multivariate analysis using conditional logistic regression mod- 180 els. Adjusted odds ratios (ORs), 95\% confidence intervals (95\% $\mathrm{CI})$ and $P$ values for trend were then estimated to assess associations adjusted for education, income, BMI, smoking, passive smoking, alcohol consumption, green tea drinking, energy intake (kcal), physical activity, and cancer in first-degree relative. 185 
The ORs were for the odds of being a hospital rather than population control. Each continuous measure of isoflavone intake was also subjected to a linear trend test.

Only for breast cancer in women were the numbers suffi-

190 ciently large to enable a cancer-specific analysis using the 2 types of matched control groups with regard to isoflavone intake. In analysis of breast cancer risk, the conditional logistic regression was further adjusted for menopausal status, oral contraceptive use, and number of children breastfed except the terms

195 included in models, which used to estimate the odds of being a hospital rather than population control.

\section{RESULTS}

Data were collected on a total of 1120 controls. Interviews were completed for 560 (response rate $90.0 \%$ ) of the 622 eligible

200 population controls who were approached to participate. The only reason for nonparticipation was refusal to provide a blood sample, which was an essential part of the overall study. Of 583 eligible hospital controls who were approached to participate, interviews were completed also for 560 with a response rate

$20596.1 \%$. For the 568 eligible cancer cases who were approached to participate, the response rate was $98.6 \%$. There were 295 breast cancer cases, 227 (132 men and 95 women) colorectal cancer cases, and 38 (21 men and 17 women) adult leukemia patients.

210 Comparisons of demographic characteristics and lifestyle factors between hospital outpatient and population controls respectively for men and women are shown in Table 1. The two control groups were remarkably similar in their exposure distributions of age (forced by matching), marital status, education,

215 income, BMI, smoking, passive smoking, alcohol intake, physical activity, tea drinking, and family history of malignancy.

Table 2 presents the adjusted ORs for isoflavone intake respectively for men and women, showing the tendency for each factor to associate independently with status as a hospital out-

220 patient rather than population control. Comparing the highest to lowest intake quartiles of isoflavone, the adjusted ORs being a hospital outpatient control were ranged 1.03-1.18 in women and 1.12-1.40 in men. The 24-point estimates of ORs ranged from 0.98 to 1.41 with all $P$ values for trend not statistically significant.

Table 3 presents ORs according to isoflavone intake for breast cancer only using the 2 types of matched control groups. Comparing the highest to lowest intake quartiles, the adjusted ORs $(95 \% \mathrm{CI})$ were $0.31(0.18,0.55)$ and $0.39(0.23,0.66)$ for

230 daidzein, $0.28(0.16,0.52)$ and $0.35(0.20,0.61)$ for genistein, $0.53(0.32,0.88)$ and $0.66(0.41,1.08)$ for glycitein, and 0.43 $(0.26,0.71)$ and $0.53(0.33,0.85)$ for total isoflavone using population and hospital outpatient controls, respectively.

\section{DISCUSSION}

This validation study, nested within 3 large case-control studies of malignancies, used separate hospital outpatient and population control groups to assess differences in the dietary isoflavone intake between the 2 control groups. Furthermore, the study evaluated if different conclusions would be reached about the effects of dietary isoflavone intake on cancer risks 240 when assessed using population and hospital outpatient controls separately. The study found that the distributions of dietary isoflavone intake in hospital outpatient controls were similar to those in population controls. In addition, we obtained similar estimates in assessing the effect of dietary isoflavone intake on 245 breast cancer in females. Therefore, our results suggest that in the context of a case-control study conducted at a Chinese hospital, regardless of whether controls were selected from hospital outpatient attendees without malignancy or drawn from population household registries covering populations in the catchment 250 area of the participating hospital, the 2 control groups appeared to have similar dietary isoflavone intake and appeared to lead to similar conclusions about effect estimates.

It is difficult to underestimate the importance of selecting appropriate controls in a case-control study. The function of 255 controls is to provide valid information on the distribution of exposure within the population at risk of becoming a case (1). Essentially, controls should have had the same opportunity and inclination to attend for their respective diagnosis as the case group did for theirs (2). Selection for appropriate groups also 260 very much depends on the health care system of the country under study. In Western countries, healthcare system is arranged in pyramid-like structure with primary care at its base. Ambulatory care and referrals are overseen by GPs who also serve as gatekeepers for access to the rest of the healthcare system 265 (22). A potential problem of hospital-based controls in Western countries is that they are not selected at random from the source population of cases and may thus be unrepresentative of the exposure distribution in that population (1).

The health care system in China is different from Western 270 countries. In terms of the place of service provision, China has inherited a largely hospital-based delivery system. Like many other health systems in Asia (including Japan and Korea), a large share of outpatient visits, even for relatively minor conditions and first-contact care, is to secondary and tertiary hos- 275 pital outpatient departments (23). There is no regulation in the present Chinese health system on the first contact of health system through primary health service institutions in particular, and there is a lack of classification of patients regarding the different levels of health service institutions. Thus, a large quantity of 280 patients with common diseases concentrates on the third-tiered health service institutions, and social health insurance programs limit coverage for providers outside the given locality (county or municipality) (23).

Therefore, patients living in cities readily visit hospitals as 285 self-referred outpatients for check-ups in China. Cultural factors and insurance arrangements lead patients to maintain a strong relationship with one particular hospital, where they receive a complete range of health care (24). Survey data in 2008 showed low levels (14\%) of community health service 
TABLE 1

Demographic and lifestyle characteristics of participants by sex and by hospital and population control status

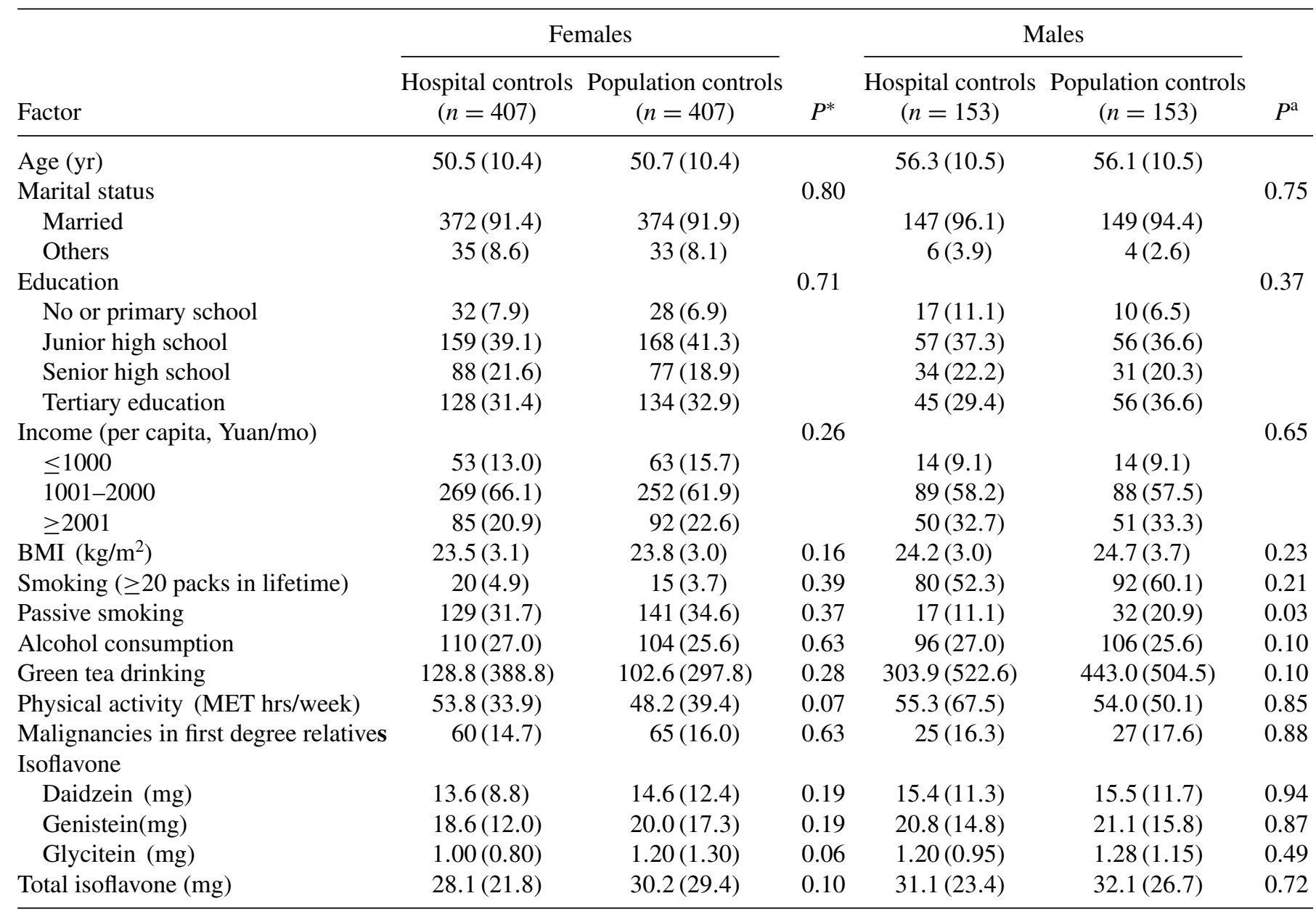

Values expressed as mean (SD) or number (\%). BMI = body mass index; MET = metabolic equivalent tasks.

${ }^{a}$ Two-sided $t$-test for continuous variables and chi-square test for categorical variables.

utilization, suggesting that community health services are not yet the first point of contact with the health system in China (25). A high proportion of the population uses hospitals for outpatient visits; therefore, hospital outpatient controls may be more valid in China than in other countries. Hospital outpatients in China are somewhat similar to ambulatory patients visiting GP clinics in Western countries.

The use of a proper, representative control population is important in reducing biases in a case-control study. The function of exposure within the population at risk of becoming a case (1-5). As there is almost never one ideal control group, some researchers believe that population registers provide the most valid way of sampling controls in a hospital case-control study (26-28), because population controls could provide information of exposure that is unaltered by associations with illness. Hospital controls, especially those with other diseases, may fail to provide an unbiased sample of the population at risk with respect to exposure status (1-5). Others suggest that generally, hospital controls should be preferred in a hospital-based case- 310 control study in view of the issues of practicability, cost, and travel time for face-to-face interviews $(29,30)$. There may also be differences in the capacity to recall and report exposures between randomly selected population members and those who are actively engaged in the health system (1). In addition, only 315 hospital controls have shown some evidence that in the event of developing the malignancy, they would be likely to attend the hospital and become a case in the study (1-3).

A few studies have compared dietary intake between the 2 types of control groups in Western countries, where none- 320 mergency access to hospital care generally depends on referral from medical practitioners in noninstitutional settings (26-29). Some studies have specifically reported differences between hospital and population controls in dietary exposure distributions (26-28). However, the findings of this investigation were 325 consistent with a smaller pilot study conducted in 1999-2000 
TABLE 2

Adjusted odds ratios and $95 \%$ confidence intervals for the odds of being a hospital control according to quartile daily intake of isoflavone by sex

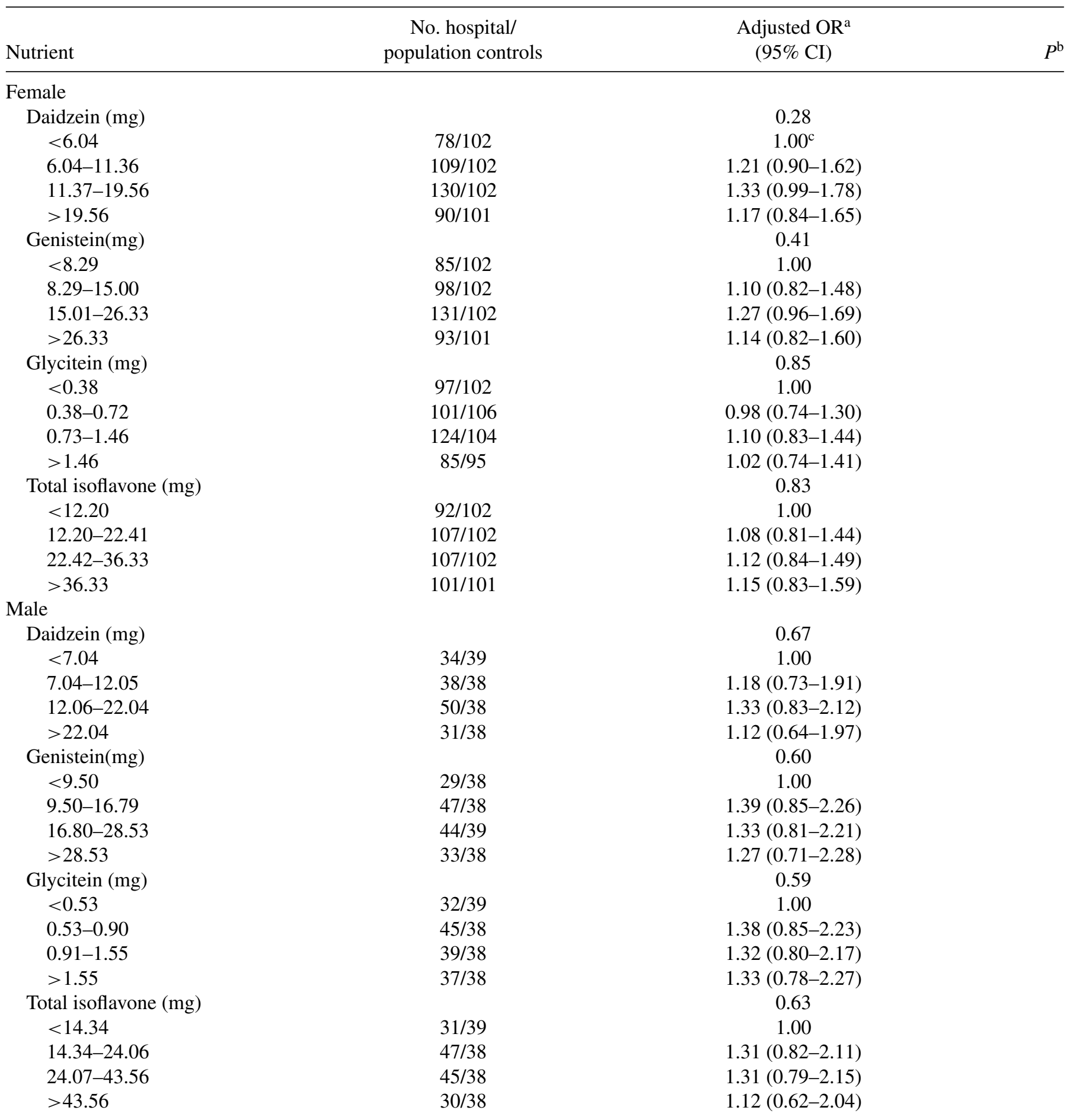

${ }^{a}$ Estimates from conditional logistic regression models included terms for education (no or primary school, junior high school, senior high school, tertiary education), income (per capita, $\leq 1000,1001-2000, \geq 2001$ Yuan/mo), body mass index now (continuous), smoking (no, yes), passive smoking (no, yes), alcohol consumption (no, yes), green tea drinking (no, yes), physical activity (weekly MET-h, continuous), energy intake (continuous, kilocalories), cancer in first-degree relative.

${ }^{\mathrm{b}}$ Two-sided test for trend across quantitative variables.

${ }^{\mathrm{c}}$ Reference group. 
TABLE 3

Adjusted odds ratios of breast cancer for isoflavone intake by hospital and population controls

\begin{tabular}{|c|c|c|c|c|}
\hline Nutrient & $\begin{array}{c}\text { No. cases/ } \\
\text { hospital controls }\end{array}$ & $\begin{array}{l}\text { Adjusted OR }{ }^{\mathrm{a}} \\
\quad(95 \% \mathrm{CI})\end{array}$ & $\begin{array}{c}\text { No. cases/ } \\
\text { population controls }\end{array}$ & $\begin{array}{l}\text { Adjusted OR }{ }^{\mathrm{a}} \\
\quad(95 \% \mathrm{CI})\end{array}$ \\
\hline \multicolumn{5}{|l|}{ Daidzein (mg) } \\
\hline $6.33-11.74$ & $101 / 84$ & $0.94(0.72-1.24)$ & $101 / 74$ & $1.03(0.78-1.36)$ \\
\hline $11.75-19.47$ & $56 / 78$ & $0.73(0.52-1.02)$ & $56 / 74$ & $0.74(0.53-1.04)$ \\
\hline \multicolumn{5}{|l|}{ Genistein(mg) } \\
\hline$<8.46$ & $115 / 69$ & 1.00 & $115 / 74$ & 1.00 \\
\hline $8.46-15.73$ & $102 / 83$ & $1.01(0.76-1.33)$ & $102 / 74$ & $1.07(0.81-1.42)$ \\
\hline $15.74-25.44$ & $60 / 76$ & $0.79(0.57-1.10)$ & $60 / 74$ & $0.79(0.57-1.10)$ \\
\hline$>25.44$ & $18 / 67$ & $00.34(0.19-0.60)$ & $18 / 73$ & $0.28(0.15-0.52)$ \\
\hline $0.74-1.46$ & $92 / 88$ & $1.07(0.78-1.47)$ & $92 / 79$ & $1.10(0.80-1.51)$ \\
\hline$>1.46$ & $26 / 53$ & $0.66(0.40-1.08)$ & $26 / 68$ & $0.55(0.33-0.92)$ \\
\hline$P$ & & 0.12 & & 0.02 \\
\hline \multicolumn{5}{|c|}{ Total isoflavone (mg) } \\
\hline$<12.49$ & $113 / 76$ & 1.00 & $113 / 74$ & 1.00 \\
\hline $12.49-23.13$ & $112 / 85$ & $1.03(0.78-1.35)$ & $112 / 74$ & $1.05(0.80-1.38)$ \\
\hline $23.14-35.12$ & $43 / 68$ & $0.73(0.50-1.06)$ & $43 / 74$ & $0.67(0.46-0.97)$ \\
\hline$>35.12$ & $27 / 66$ & $0.52(0.33-0.85)$ & $27 / 73$ & $0.45(0.27-0.75)$ \\
\hline$P$ & & 0.02 & & $<0.01$ \\
\hline
\end{tabular}

${ }^{a}$ Estimates from conditional logistic regression models included terms for education (no or primary school, junior high school, senior high school, tertiary education), income (per capita, $\leq 1000,1001-2000, \geq 2001$ Yuan/mo), household size (continuous), body mass index 5 yr ago (continuous), smoking (no, yes), passive smoking (no, yes), alcohol consumption (no, yes), green tea drinking (no, yes), physical activity (weekly MET-h, continuous), energy intake (continuous, kilocalories), cancer in first-degree relative, menopausal status, oral contraceptive use, and number of children breastfed.

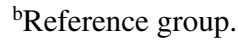

${ }^{\mathrm{c}}$ Two-sided test for trend across quantitative variables.

by our research team (15), as well as with another case-control study that compared noncancer outpatients' food consumption with population controls in Japan which has similar health care system as China (30). The discrepancies between this study and other studies conducted in Western countries might also due to that our study used outpatients as hospital controls, other studies conducted in Western countries used inpatients as hospital controls.

In Asian countries, cancer incidences are generally lower than in Western populations. An association between the high intakes of soy foods in Asian countries and the low breast, prostate, endometrial, and colorectal cancer risk has been suggested. Amongst other things, isoflavone are discussed as the soy ingredients responsible for the biological effect (31). In this study, the inverse associations of breast cancer were observed regardless of which control series was used to represent the un- derlying exposure of the highest quartiles isoflavone intake. The results were consistent with the cancer-inhibitory effects of soy isoflavone suggested from a meta-analysis of 18 epidemiologic studies that found an overall inverse association between soy intake and breast cancer risk (32), a large prospective cohort study conducted among Chinese women that suggests that an increase in soy food intake can reduce the risk of breast cancer (33), our previous finding that increasing the quantity of isoflavone 350 intake reduces the risk of breast cancer in Chinese women (8), as well as with a study that suggested that soy isoflavone intake was associated with a significant reduced risk of breast cancer incidence in Asian populations (34).

The effects of isoflavone intake estimated in this study sug- 355 gest that hospital outpatient controls performed little different from population controls in the assessment of dietary isoflavone intake. To our knowledge, the study is the first to investigate 
differences in dietary isoflavone intake between population and 360 hospital outpatient controls in China. The strengths of the study were the interviews in all of controls were conducted by a single investigator to avoid inter-interviewer variation. As far as potential sources of biases are concerned, selection bias was likely to be minimal in view of the high recruitment rate $(96.1 \%$

365 for hospital outpatient control, $90.0 \%$ for population controls), our systematic recruitment procedure, and as evidenced by the remarkably similar distributions of demographic characteristics and lifestyle factors between the 2 control groups (7). The study attracted participation from most $(98.6 \%)$ cancer patients ap-

370 proached. Hospital records were reviewed daily and all cases had been accounted for. The majority of cases were newly diagnosed and interviewed within 3 mo after diagnosis, and responses were elicited on habitual dietary intake by using a "reference" period before diagnosis. The recruitment and iden-

375 tification procedures ensured that ascertainment of cases was maximized and complete. Extensive information on soy foods intake (the questionnaire includes virtually major soy foods that are commonly consumed in China) and dietary patterns was obtained using a validated and reliable instrument specifically

380 developed for the adult Chinese population (16-18).

The study was carefully adjusted for a wide range of dietary and nondietary factors that are potential confounders of dietary isoflavone intake and breast cancer association, such as the adjustment of caloric intake, physical activity, and green

385 tea consumption; the latter were shown to be protective factors against breast cancer (35). Furthermore, the bioavailability of self-reported usual intake of soy isoflavone as determined from the FFQ has been documented in the literature. There were strong correlations among plasma concentrations, urinary ex-

390 cretion of isoflavone, and self-reported soy intake (36-38), suggesting that the usual soy intake by participants and isoflavone estimates as derived from the FFQ should be reasonably reliable for subsequent analysis. A recent isoflavone database from the USDA was used to estimate isoflavone intake, which had

395 been developed specifically for Chinese immigrants in America (39). Moreover, our participants had relatively large isoflavone intake with substantial variation, making them suitable for the investigation of this association.

The major limitation of this study is that it was based in a sin-

400 gle hospital, a multi-center study may be needed to confirm our results. However, the study hospital, which is one of the largest hospitals in Shenyang, attracts most patients from catchment area. Therefore, our results are fairly representative of urban outpatient. Another limitation of this study is that the case-control 405 design may have introduced certain sources of bias (e.g., recall bias), nevertheless, a large population-based prospective cohort study has confirmed our results (33).

In conclusion, with respect to dietary isoflavone intake included in the present study, our results suggest that hospital 410 outpatient controls performed little different from population controls. For the effect estimates for cancer case groups, using hospital outpatient controls were similar to those using pop- ulation controls. Therefore, even though some concerns exist, hospital outpatients provide a satisfactory control group in the assessment of dietary isoflavone intake in hospital-based case- 415 control study in the Chinese hospital setting. In addition, dietary isoflavone intake may offer some health advantages such as breast cancer protection.

\section{ACKNOWLEDGMENTS}

This work was supported by the National Health and Medical 420 Research Council of Australia project grant (APP ID 572542). Lin Li was supported by the Australian Postgraduate Award and the University of Western Australia Establishment Award. The authors acknowledge with gratitude the participation of patients, outpatients, and community residents in Shenyang. We are grateful for the collaboration received from the participating hospital and the staff, and from community councils. In particular, we would like to thank Professor Liu Yun-Peng and Dr. Shi Jing of the First Hospital of China Medical University, for their kind assistance.

\section{REFERENCES}

1. Wacholder S, Silverman DT, McLaughlin JK, et al. : Selection of controls in case-control studies. I principles; II types of controls; III design options. Am J Epidemiol 135, 1019-1050, 1992.

2. Rothman KJ, Greenland S, and Lash TL: Case-control studies. In Modern 435 epidemiology, 3rd ed., Rothman KJ, Greenland S, and Lash TL (eds.). Philadelphia: Wolters Kluwer Health/Lippincott Williams \& Wilkins, 2008, pp. 111-127.

3. Miettinen OS: Theoretical Epidemiology: Principles of Occurrence Research in Medicine. John Wiley \& Sons, New York, 1985.

4. Miettinen OS: The concept of secondary base. J Clin Epidemiol 43, 1017-1020, 1990.

5. Grimes DA and Schulz KF: Compared to what? Finding controls for casecontrol studies. Lancet 365, 1429-1433, 2005.

6. Hu S, Tang S, Liu Y, Zhao Y, Escobar ML, et al.: Reform of how health care 445 is paid for in China: challenges and opportunities. Lancet 372, 1846-1853, 2008.

7. Li L, Zhang M, and Holman CDJ: Population versus hospital controls for case-control studies on cancers in Chinese hospitals. BMC Med Res Methodol 11, 167, 2011. doi:10.1186/1471-2288-11-167

8. Zhang M, Liu X, and Holman CDJ: Effect of dietary intake of isoflavone on the estrogen and progesterone receptor status of breast cancer. Nutr Cancer 62, 765-773, 2010.

9. Shu XO, Zheng Y, Cai H, Gu K, Chen Z, et al.: Soy Food intake and breast cancer survival. JAMA 302, 2437-2443, 2009.

10. Budhathoki S, Joshi AM, Ohnaka K, Yin G, Toyomura K, et al.: Soy food and isoflavone intake and colorectal cancer risk: the Fukuoka Colorectal Cancer Study. Scand J Gastroenterol 46, 165-172, 2011.

11. Chang SC, Rashid A, Gao YT, Andreotti G, Shen MC, et al.: Polymorphism of genes related to insulin sensitivity and the risk of biliary tract cancer and 460 biliary stone: a population-based case-control study in Shanghai, China. Carcinogenesis 29, 944-948, 2008.

12. Hsing AW, Sakoda LC, Rashid A, Andreotti G, Chen J, et al.: Variants in inflammation genes and the risk of biliary tract cancers and stones: a population-based study in China. Cancer Res 68, 6442-6452, 2008.

13. Porta M, Greenland S, and Last JM: A dictionary of epidemiology, 5th ed. Oxford University Press, New York, 2008. 
14. Zhang M, Holman CDJ, Huang JP, and Xie X: Green tea and the prevention of breast cancer: a case-control study in Southeast China. Carcinogenesis 28, 1074-1078, 2007.

15. Zhang M, Yang ZY, Binns CW, and Lee AH: Diet and ovarian cancer risk: a case-control study in China. Brit J Cancer 86, 712-717, 2002.

16. Ji BT, Chow WH, Yang G, Mclaughlin JK, Zheng W, et al.: Dietary habits and stomach cancer in Shanghai, China. Int J Cancer 76, 659-664, 1998.

17. Zhang M, Binns CW, and Lee AH: A quantitative food frequency questionnaire for women in southeast China: Development and reproducibility. Asia Pac J Public Health 17, 29-35, 2005.

18. Jian L, Binns CW, and Lee AH. Validity of a food-frequency questionnaire for elderly men in southeast China. Public Health Nutr 9, 928-933, 2006.

19. Whitemore AS, Wu-Willians AH, Lee M, Lee M, Zheng S, et al.: Diet, physical activity and colorectal cancer among Chinese in North American and China. J Natl Cancer Inst 82, 915-926, 1990.

20. Institute of Nutrition and Food Hygiene, Chinese Academiy of Preventive Medicine: Food Composition Table (National Representative Values), 1st ed. People's Health Press, Beijing, China, 1999.

21. United States Department of Agriculture: USDA Database for the Isoflavone Content of Selected Foods, 2008. Retrieve drom http://www.ars. usda.gov/nutrientdata/isoflav

22. Brzezinski N: Does a universal health care system cause a decrease in quality of care?: A comparison of American and British quality in primary care. IJAPA 6, 10, 2009. doi:10.5580/1fb0

23. Xu J, Wang W, Li Y, et al. Analysis of factors influencing the outpatient workload at Chinese health centres. BMC Health Serv Res 10, 151, 2010.

24. Wang J, Kushner K, Frey JJ, Du XP, and Qian N: Primary care reform in the Peoples' Republic of China: implications for training family physicians for the world's largest country. Fam Med 39, 639-643, 2007.

25. Bhattacharyya O, Delu Y, Wong ST, and Bowen C: Evolution of primary care in China 1997-2009. Health Policy 100, 174-180, 2011.

26. Almendingen K, Hofstad B, Trygg K, Hoff G, Hussain A, et al. : Current diet and colorectal adenomas: a case-control study including different sets of traditionally chosen control groups. Eur J Cancer Prev 10, 395-406, 2001.

27. Amadori D, Nanni O, Ricci M, Falcini F, Decarli A, et al.: Hospital versus population controls in a retrospective study on diet and stomach cancer. Eur J Public Health 5, 209-214, 1995.
28. Malagoli C, Vinceti M, Pellacani G, Sieri S, Krogh V, et al. : Diet and melanoma risk: effects of choice of hospital versus population controls. Tumori 94, 669-673, 2008.

29. GonzÁLez CA, Torrent M, Agudo A, and Riboli E: Hospital versus neighbourhood controls in the assessment of dietary risk factors. Int J Epidemiol 510 19, 354-361, 1990.

30. Inoue M, Tajima K, Hirose K, Hamajima N, Takezaki T, et al.: Epidemiological features of first-visit outpatients in Japan: comparison with general population and variation by sex, age, and season. J Clin Epidemiol 50, 69-77, 1997.

31. Andres S, Abraham K, Appel KE, and Lampen A: Risks and benefits of dietary isoflavone for cancer. Crit Rev Toxicol 41, 463-506, 2011.

32. Trock BJ, Hilakivi-Clarke L, and Clarke R: Meta-analysis of soy intake and breast cancer risk. J Natl Cancer Inst 98, 459-471, 2006.

33. Lee SA, Shu XO, Li H, Yang G, Cai H, et al.: Adolescent and adult soy food 520 intake and breast cancer risk: results from the Shanghai Women's Health Study. Am J Clin Nutr 89, 1920-1926, 2009.

34. Dong JY and Qin LQ: Soy isoflavone consumption and risk of breast cancer incidence or recurrence: a meta-analysis of prospective studies. Breast Cancer Res Treat 125, 315-323, 2011.

35. Sun CL, Yuan JM, Koh WP, and Yu MC: Green tea, black tea and breast cancer risk: a meta-analysis of epidemiological studies. Carcinogenesis 27, 1310-1315, 2006

36. Maskarinec G, Singh S, Meng L, and Franke AA: Dietary soy intake and urinary isoflavone excretion among women from a multiethnic population. Cancer Epidemiol Biomark Prev 7, 613-619, 1998.

37. Verkasalo PK, Appleby PN, Allen NE, Davey G, Adlercreutz H, et al.: Soya intake and plasma concentrations of daidzein and genistein: validity of dietary assessment among eighty British women (Oxford arm of the European Prospective Investigation into Cancer and Nutrition). Br J Nutr 535 86, 415-421, 2001.

38. Wu AH, Yu MC, Tseng CC, Twaddle NC, and Doerge DR: Plasma soflavone levels versus self-reported soy isoflavone levels in Asian-American women in Los Angeles County. Carcinogenesis 25, 77-81, 2004.

39. Horn-Ross PL, Barnes S, Lee M, Coward L, Mandel JE, et al.: As- 540 sessing phytoestrogen exposure in epidemiologic studies: development of a database (United States). Cancer Causes Control 11, 289-298, 2000. 